\title{
Au pays maya des lacs et des marais. Recherches archéologiques et géographiques (1999-2001)
}

Marie-Charlotte Arnauld, Ernesto Arredondo, Jean-Michel Carozza, Véronique Breuil-Martínez, Mélanie Forné, Didier Galop, Laura Gamez, Véronique Gervais, Diego Guerra, Marco Antonio Leal, Éva Lemonnier, Salvador López, Raquel Macario, Jean-Paul Métailié, Dominique Michelet, Erick Ponciano, Martin Rangel, Gilles Selleron, Marie Soubelet, Nadine Tisnerat, Sébastien Lacombe, Enrique Monterroso et Tristan Saint-Dizier

\section{OpenEdition} Journals

Édition électronique

URL : http://journals.openedition.org/jsa/11206

DOI : 10.4000/jsa. 11206

ISSN : 1957-7842

Éditeur

Société des américanistes

Édition imprimée

Date de publication : 1 janvier 2001

Pagination : 283-302

ISSN : 0037-9174

Référence électronique

Marie-Charlotte Arnauld, Ernesto Arredondo, Jean-Michel Carozza, Véronique Breuil-Martínez, Mélanie Forné, Didier Galop, Laura Gamez, Véronique Gervais, Diego Guerra, Marco Antonio Leal, Éva Lemonnier, Salvador López, Raquel Macario, Jean-Paul Métailié, Dominique Michelet, Erick Ponciano, Martin Rangel, Gilles Selleron, Marie Soubelet, Nadine Tisnerat, Sébastien Lacombe, Enrique Monterroso et Tristan Saint-Dizier, « Au pays maya des lacs et des marais. Recherches archéologiques et géographiques (1999-2001) », Journal de la société des américanistes [En ligne], 87 | 2001, mis en ligne le 18 juillet 2010, consulté le 03 mai 2019. URL : http:// journals.openedition.org/jsa/11206 ; DOI : 10.4000/jsa.11206 


\section{AU PAYS MAYA DES LACS ET DES MARAIS RECHERCHES ARCHÉOLOGIQUES ET GÉOGRAPHIQUES}

(1999-2001)

Marie-Charlotte Arnauld, Ernesto Arredondo, Jean-Michel Carozza, Véronique Breull-Martínez, Mélanie Forné, Didier Galop, Laura Gamez, Véronique Gervais, Diego Guerra, Sébastien Lacombe, Marco Antonio Leal, Éva Lemonnier, Salvador López, Raquel Macario, Jean-Paul MÉtailié, Dominique Michelet, Enrique Monterroso, Erick Ponciano, Martin Rangel, Tristan Saint-Dizier, Gilles Selleron, Marie Soubelet, Nadine Tisnerat*

Les sociétés mayas des hautes terres ont connu un déclin culturel marqué à la fin du Préclassique (vers 100-250 apr. J. C.) au moment où, pour la plupart, les cités mayas des basses terres prenaient leur essor au début du Classique (250-300 apr. J. C.). Environ 600 ans plus tard, celles du Petén (Guatemala) étaient presque toutes abandonnées, tandis que celles des basses terres du nord (Mexique) connaissaient leur apogée jusqu'au début du Postclassique. Comprendre ces histoires culturelles marquées de ruptures exige de reconstruire des séquences locales, indépendamment les unes des autres. À nos recherches à Balamkú (Campeche, Mexique; Michelet et al. 1997, 1998 ; voir aussi Arnauld et al. 1999) succèdent celles de La Joyanca (Petén, Guatemala). La question générale peut se formuler ainsi : quels caractères de l'organisation socio-politique et économique peuvent être isolés archéologiquement pour telle et telle cité, qui permettent de comprendre tantôt l'effondrement, tantôt la récupération, survenus lors de crises régionales ? En termes géographiques, il s'agit d'analyser l'évolution d'anthropo-systèmes formés en forêt tropicale humide de mousson, soit une des rares zones de ce type sur la planète où s'est développée dans le passé une civilisation puissante, agricole et urbaine sur une longue durée (le Cambodge en est un des seuls autres exemples). Cette problématique générale est celle de deux grands programmes de recherche récemment développés dans l'ouest du Petén, au Petexbatún et à Piedras Negras (Demarest 1997 ; Escobedo \& Houston 1999), au sud et au sud-ouest de La Joyanca.

Le projet Petén nord-ouest (PNO)-La Joyanca a pour objet l'étude d'une communauté socio-politique de rang moyen, d'époque classique (250-850 apr. J. C.), située

* Archéologie des Amériques (UMR 8096, Nanterre), GEODE Géographie de l'Environnement (UMR 5602, Toulouse), Chrono-écologie du Quaternaire (UMR 9946, Besançon), Laboratoire des Sciences du Climat et de l'Environnement (UMR 1572, Gif-sur-Yvette), CEREG Centre d'Études et de Recherches écogéographiques (UMR 7007, Strasbourg), université de San Carlos, université del Valle (Guatemala).

Journal de la Société des Américanistes 2001, 87, p. 283 à 302. Copyright @ Société des Américanistes. 


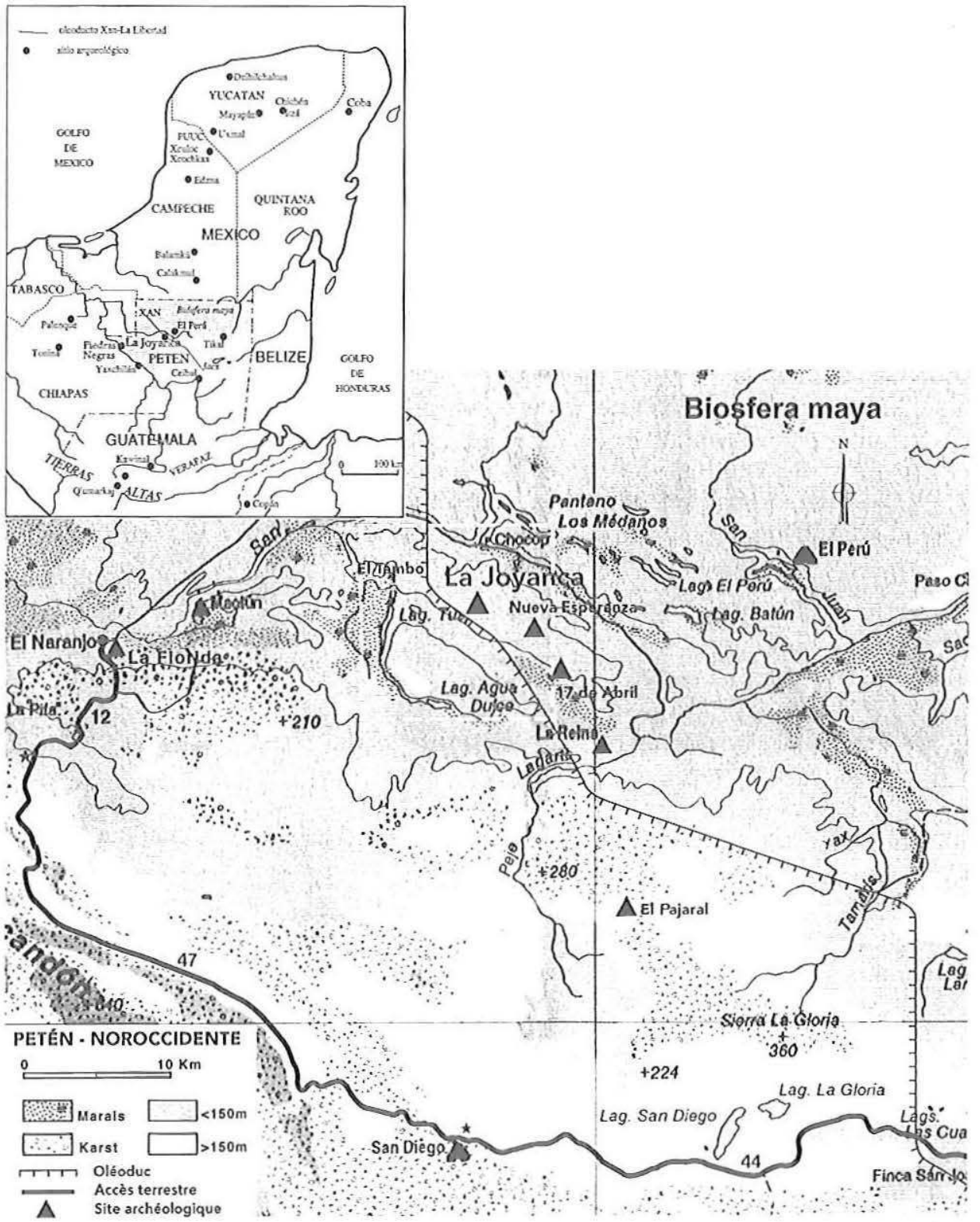

Fig. 1. - Carte de la région de La Joyanca, Petén nord-ouest, Guatemala

dans une région des basses terres jusqu'à maintenant quasiment inconnue du point de vue archéologique, le Petén nord-ouest (Figure 1). Cette région se définit par la présence de marais étendus à des altitudes comprises entre 50 et $200 \mathrm{~m}$ et d'un réseau serré d'affluents des grands fleuves du Tabasco (Mexique) qui se jettent dans le golfe du Mexique. Ce projet est d'un type particulier, d'une part parce qu'il conjugue une 
approche archéologique et une approche géographique, cherchant à restituer l'histoire des hommes en même temps que l'histoire du milieu, d'autre part parce que l'initiative de cette recherche puis son financement sont le fait d'une compagnie pétrolière, qui opère depuis longtemps dans le nord-ouest du Petén. Cette région semble bien avoir été inaccessible par les moyens habituels des institutions de recherche (encore qu'il y ait eu une tentative vers 1987 de la part de l'université de Pennsylvanie) : du moins en 1997, elle demeurait quasiment inexplorée, ce qui en soi constituait un réel défi étant donné que le restant de la péninsule du Yucatán a fait l'objet de très nombreuses études de sites depuis les années vingt.

Le choix du site n'est pas le fait des chercheurs. La Joyanca a été découvert en 1994 par les archéologues de la compagnie Basic, Marco Antonio Leal et Salvador López, lors des études d'impact réalisées en vue de la construction de l'oléoduc Xan-La Libertad. Le tracé en a été légèrement modifié pour respecter la zone protégée définie en accord avec l'Institut d'anthropologie du Guatemala. Deux gardiens y ont été placés et le plan préliminaire du site a été levé en 1996. Notre première mission d'évaluation en vue d'un éventuel programme archéologique et géographique remonte à l'été 1997. Trois saisons de terrain, de 10 à 15 semaines chacune, ont eu lieu depuis $1999^{1}$ (Arnauld \& Morales 1999 ; Arnauld, Ponciano \& Breuil-Martinez 2000).

La Joyanca se situe donc en bordure d'un oléoduc, dont la «brèche » en forêt a plus ou moins servi de voie de pénétration pour des colons venus des hautes terres du Guatemala depuis 1993. Ils y ont trouvé des terres vierges depuis des siècles. Les acajous en ont été extraits plus ou moins clandestinement au $\mathrm{xx}^{\mathrm{e}}$ siècle, et envoyés au Mexique par le fleuve San Pedro Mártir qu'on atteint en moins de deux heures par le plateau de La Joyanca et des voies d'eau. Le San Pedro était au XIX ${ }^{\mathrm{e}}$ siècle et au début du $\mathrm{xx}^{\mathrm{e}}$ siècle la voie de pénétration habituelle du Petén depuis « l'extérieur » (les voies d'accès actuelles par l'est et par le sud du Petén sont très récentes) : Maler, Maudslay, Charnay et bien d'autres l'ont empruntée. Au Xvi ${ }^{\mathrm{e}}$ siècle, c'est aussi par cette voie que Cortés a atteint le Petén depuis le Mexique central et le Tabasco. Mais le site de La Joyanca est situé trop loin du San Pedro et est trop diffícile d'accès pour susciter a priori une problématique portant sur les échanges préhispaniques, commerciaux, culturels ou autres ${ }^{2}$.

L'intérêt du site nous a paru, lors des missions préliminaires, relever d'autres caractéristiques. D'une part, ses dimensions moyennes permettent d'y travailler une proportion substantielle de ses composants, ce qui garantit une bonne représentativité des données obtenues quant à sa " culture matérielle», par quoi on entend habituellement, dans les basses terres mayas, l'architecture, les expressions rituelles telles que sépultures et offrandes, l'évolution céramique qui reflète une chronologie relative de l'occupation (complétée de datations absolues radiocarbone), l'équipement lithique, etc. D'autre part, le degré de complexité moyen de son "urbanisme» oụ, plus modestement, des structures de son habitat, autorise d'y tenter l'étude diachronique de la communauté hiérarchisée qui s'est formée là, dès le premier millénaire avant J. C. sur le plateau dominant le San Pedro, les marais et les lacs. Comment s'est formée la hiérarchie de son habitat en termes de hiérarchie sociale et d'organisation politique et que signifie-t-elle à l'apogée de son occupation (600-850) ? Comment comprendre son centre monumental en termes de " gouvernement local » ? Quelles furent ses relations d'autonomie ou de dépendance vis-à-vis des centres, moyens et grands, connus ou à 
connaître dans la région? Cette " petite cité » fut-elle abandonnée comme le restant des basses terres centrales au IXe siècle et pourquoi ? Telles sont les composantes de la problématique socio-politique que nous avons formulée, une fois admis que méritait d'être relevé le double défi d'une recherche « sponsorisée ", à mener dans une région inconnue, difficile d'accès et à peine colonisée.

Le troisième caractère de La Joyanca digne d'intérêt relève de son implantation et des possibilités qu'elle offre : le plateau que le site occupe forme un territoire et un terroir potentiels bien délimités au-dessus des marais et des lacs. On a là un contexte favorable à l'étude des agrosystèmes mis en place par les Mayas à leurs différents stades de développement. Quel en a été l'impact au niveau de la végétation, des sols et de la dynamique du paléo-karst, construit dans un bas plateau de calcaires tendres ? Quel a pu être le rôle des variations climatiques dans un milieu à la fois très humide et soumis à des périodes de sécheresse importantes? Sa colonisation actuelle tout à fait récente n'a pas encore gravement modifié le milieu. Il s'agit donc de restituer l'histoire du milieu forestier et des paysages, de vérifier si possible l'intensité des pratiques agricoles et d'en donner une représentation spatiale, tant pour l'époque classique (250-850 apr. J. C.) que pour le présent, car il n'y a pas d'histoire de l'environnement qui ne s'appuie sur une connaissance solide du milieu actuel.

On présentera maintenant les résultats préliminaires du programme, tels qu'ils sont formulés quelques semaines après la fin de la troisième campagne de terrain (5 mai 2001), en indiquant brièvement la nature des données qui les fondent et leur mode d'obtention (reconnaissances de surface, décapages, sondages, fouilles extensives...). On traitera successivement du contexte régional dans lequel s'insérait la cité de La Joyanca, dont le statut politique a probablement varié, de la formation et de l'évolution de la communauté qu'elle constituait, de son centre religieux et politique et, enfin, de son habitat domestique inscrit dans un environnement assez modifié par l'homme.

\section{LE CONTEXTE RÉGIONAL}

Plusieurs reconnaissances régionales ont été réalisées par M. A. Leal et S. López, non seulement à l'échelle du plateau de La Joyanca et des plateaux voisins (environ $30 \mathrm{~km}^{2}$ chacun), mais aussi à l'échelle plus vaste d'un secteur d'environ $600 \mathrm{~km}^{2}$ défini à la fois par la géomorphologie et l'archéologie (Figure 1), soit grosso modo un triangle incluant les marais et les lacs liés au Río San Pedro, situé au nord des plissements de la Sierra del Lacandón, dont le sommet sud est le site archéologique de El Pajaral et dont la base nord passe à environ $10 \mathrm{~km}$ au nord de El Perú (est) et de Mactún. Ces grands sites archéologiques, connus avant 1997, constituaient peut-être une structure politique régionale dans laquelle s'inscrivait La Joyanca.

À l'échelle des plateaux, entre le lac Tuspán, le lac Agua Dulce et le Río San Pedro, l'habitat maya ancien semble avoir été constitué de petits groupes dispersés, d'un ou deux « patios » intégrant chacun quatre ou cinq petits bâtiments domestiques; ils sont tous situés en bordure de plateaux, ayant ainsi un accès direct à l'eau des marais ou des lacs, et probablement aux bonnes terres humides des rives, où deux récoltes annuelles sont garanties. Ce serait le niveau le plus bas d'une hiérarchie régionale de l'habitat, qui en compterait six. Le cinquième niveau hiérarchique de l'habitat correspond à ce 
que nous appelons les «grands groupes résidentiels » (GGR), formés de monticules étroits et longs, souvent de plus d'un mètre de hauteur, bordant sur quatre côtés une petite place, ou patio assez vaste, le tout atteignant $50 \mathrm{~m}$ de côté ; quand les angles du patio sont fermés (ou paraissent tels), il s'agit de « quadrilatères ». Les GGR sont peu nombreux sur les plateaux, et distants les uns des autres, mais deux sites proches de La Joyanca (4 à $5 \mathrm{~km}$ ) en regroupent cependant plusieurs à moins de $300 \mathrm{~m}$ de distance les uns des autres : La Flor de la Nueva Esperanza et 17 de Abril, noms des communautés de colons qui se sont implantées en 1998 justement sur ces sites. La distribution des GGR sur les plateaux et, on le verra, dans l'établissement même de La Joyanca, reflète probablement la structure socio-économique fondamentale des plateaux, celle qu'il convient d'étudier et de restituer en priorité. La forêt et, parfois, l'hostilité des colons rendent cependant le travail difficile et lent.

Un quatrième niveau hiérarchique dans l'habitat correspond provisoirement à un seul site, El Tambo (Groupe R), qui présente un véritable petit centre religieux et politique, avec pyramides, terrain de jeu de balle ouvert (donc du Classique ancien ?), et bâtiments longs disposés de façon orthogonale. À l'échelle régionale, on aborde le troisième niveau hiérarchique, qui est en principe celui de La Joyanca. Du côté des rives sud du San Pedro (sur les rives nord, nos reconnaissances ont commencé en avril 2001), Mactún et La Reina sont caractérisés par de hautes pyramides, mais seraient dénués de monuments sculptés et de terrains de jeu de balle. Plus au sud, à la limite des deux régions géomorphologiques citées, Leal et López ont réussi à relocaliser El Pajaral, un site " perdu » depuis les années cinquante (Graham \& Von Euw 1982). Situé en partie sur un éperon rocheux dominant un marais, El Pajaral comporte un centre politique et religieux très compact, avec de hautes pyramides, mais sans jeu de balle ; les complexes résidentiels peuvent être assimilés à la classe GGR, encore que la topographie accidentée du site en réduise la surface. El Pajaral était doté d'une série de stèles sculptées représentant des gouvernants, et portant des textes glyphiques ; il n'en reste que deux complètes, outre les fragments d'un nombre indéterminé de stèles pillées. David Stuart, du Corpus des inscriptions mayas de l'université de Harvard, en a commencé l'inventaire et le déchiffrement en mars 2001. Au moins une stèle remonte au Classique ancien (250-600 apr. J. C.), les autres sont du Classique récent (600-850); un toponyme, Hixwitz, semble y être mentionné. Il est possible que El Pajaral ait constitué le centre recteur de la région sud du San Pedro, au moins durant un moment du Classique récent ; il marquerait à ce titre notre deuxième niveau hiérarchique de l'habitat ancien.

Le premier niveau hiérarchique correspond sans conteste au site de El Perú, situé à $20 \mathrm{~km}$ à l'est de La Joyanca sur une éminence dominant un lac et un affluent nord du San Pedro. El Perú est une des grandes cités mayas classiques des basses terres, dotée en principe de son propre emblème-glyphe dès le Classique ancien (8.18.0.0.0, Mathews 1985 ; voir Berlin 1958). Ses sculptures, peut-être aussi sa céramique, sont de grande qualité (Graham 1988 ; Reents \& Bishop 1987, pp. 783-785 ; Robicsek \& Hales 1981) et les inscriptions indiquent que ses gouvernants reconnaissaient la souveraineté de Calakmul (Simon \& Grube 1995). El Perú n'a cependant jamais été fouillé, ni même décrit; nous avons constaté sur place que la maçonnerie des édifices visibles est de pierres de parement finement taillées et que le terrain de jeu de balle est du type fermé. 
La Joyanca dépendait sans aucun doute de El Perú, mais peut-être aussi de El Pajaral. Dénuée de jeu de balle, la Place Principale de ce centre politico-religieux comporte deux temples-pyramides de 10-12 $\mathrm{m}$ de hauteur; un bâtiment fort long $(65 \mathrm{~m})$ plutôt public, un édifice à trois pièces au nord et deux hautes plates-formes à l'est et au sud (Figures 2 et 3). L'unique stèle trouvée sur le site en février 2001 (Figure 6) se trouve hors de cette place, dans le plus grand et le plus complexe des huit GGR, le Groupe Guacamaya ; dénuée d'image, elle ne porte que deux inscriptions glyphiques sur les chants. David Stuart y lit une date du Classique ancien, 9.2.10.0.0, soit 485 apr. J. C., l'une des plus anciennes dates calendaires mayas dans l'ouest des basses terres (Mathews 1985). Il y repère peut-être là encore le toponyme Hixwitz, ce qui tendrait à suggérer que La Joyanca appartenait au réseau politique sud de El Pajaral, au moins vers la fin du Classique ancien. Nous n'avons aucun indice permettant de penser que d'autres stèles aient été érigées postérieurement; cela tend à indiquer que La Joyanca a perdu le statut régional dont elle jouissait au Classique ancien. Il est remarquable que cette unique stèle, associée à des monolithes de calcaires non sculptés ayant servi d'autels (l'abondance des tessons de céramique alentour l'atteste), soit située dans le Groupe Guacamaya, le groupe résidentiel le plus complexe et peut-être le plus ancien du site, mais qui est séparé de la Place Principale. Autant le centre de Pajaral se caractérise par une forte intégration, doté en outre de la plus grande stèle du site, autant la disjonction entre la Place Principale et le Groupe Guacamaya est un trait marquant à La Joyanca, assez peu fréquent dans les centres du Petén.

\section{LA FORMATION DE LA « CITÉ » LA JOYANCA}

L'établissement de La Joyanca est peu intégré, assez peu dense, nettement hiérarchisé dans ses composants et, bien qu'il comporte un centre entouré d'une zone résidentielle qui laisse place à la périphérie rurale, le modèle concentrique s'y applique mal. En fait, la Place Principale est située sur le rebord sud du plateau, ainsi que le plus grand des ensembles résidentiels à patios multiples, le Groupe Guacamaya, dit de classe I. Au nord, au nord-est et au nord-ouest sont dispersés sept autres grands groupes résidentiels de classe II ou GGR (Venado, Loro Real, etc.), presque tous à patios multiples aussi, chacun étant distant du voisin de 100 à $150 \mathrm{~m}$. Des monticules bas souvent groupés par paires les entourent généralement ; le plus grand groupement de tels vestiges d'habitations modestes est associé au Groupe Guacamaya (60 unités, de classe IIIA). Enfin, des groupes de classe IIIB comportent des patios domestiques de dimensions moyennes. Nos fouilles ( $c f$. infra) ont montré que les maisons des groupes de classes I et II avaient des murs et un toit voûté maçonnés (du moins dans les grands patios) ; mais nous avons constaté en 2001 que même certains édifices de patios de classe IIIB pouvaient être voûtés. Au total, jusqu'à maintenant, 412 structures ont été repérées sur environ 160 ha, soit une densité de 2,6 structures à l'hectare ou $260 / \mathrm{km}^{2}$, et de $300 / \mathrm{km}^{2}$ strictement dans la zone résidentielle (en incluant Guacamaya : 294 structures sur 120 ha). Ce sont là des densités équivalentes à celles des sites des basses terres centrales (Culbert \& Rice 1990, Drennan 1988, Nondédéo 2001, p. 181). Mais il faudrait les corriger à la baisse, par l'évaluation de la contemporanéité 


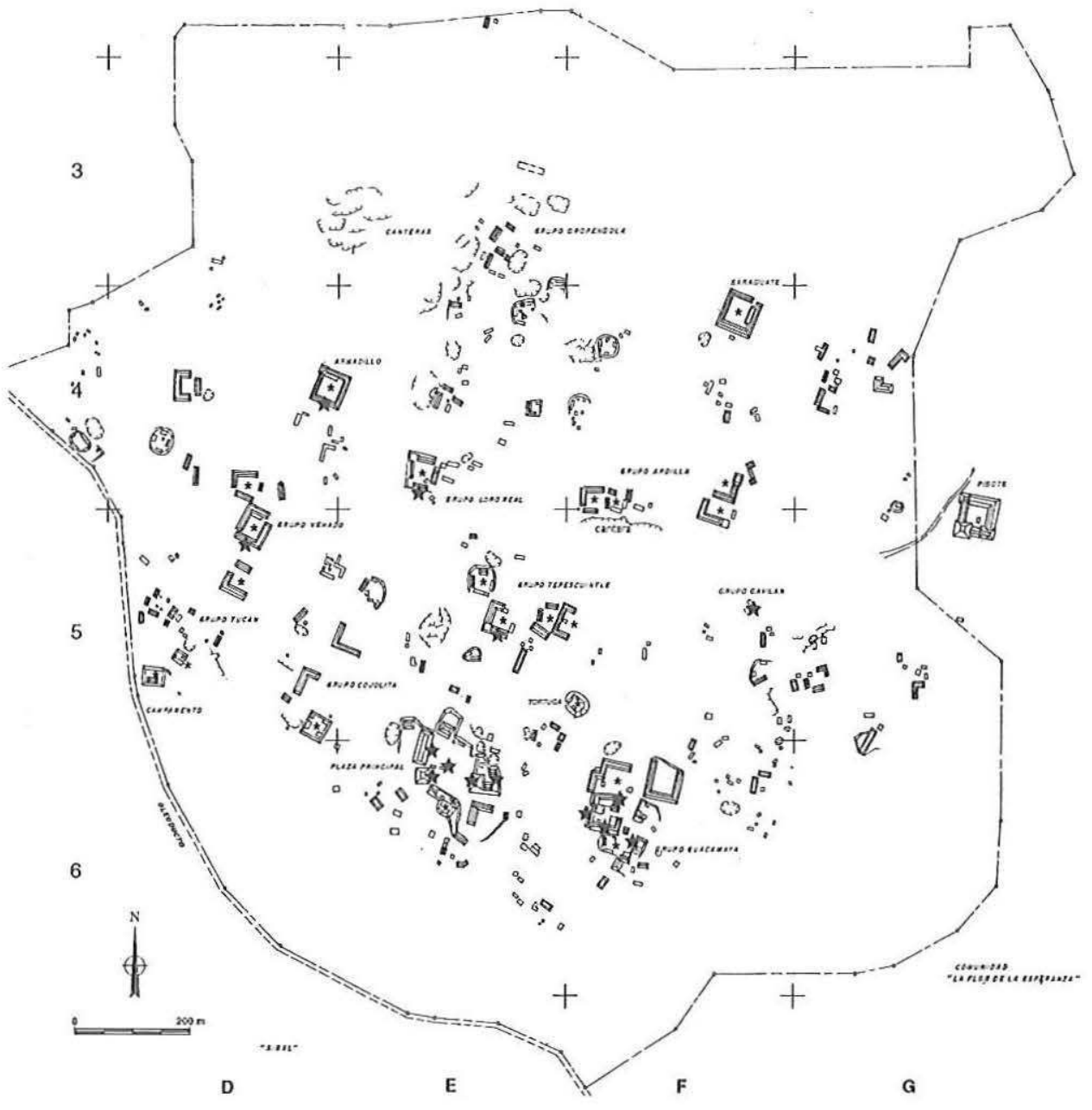

Projet PNO - La Joyanca

Plan du site

Les étoiles $(\boldsymbol{k})$ localisent les fouiles d'architecture,

le symbole (*) remplacement des sondages réalisés en 1999-2001.

Fig. 2. - Plan préliminaire de La Joyanca (1996, IDAEH \& Basic Resources)

des structures, et à la hausse, par celle de la fréquence des structures non visibles en surface.

Pour la chronologie de cet établissement, nous disposons de l'analyse céramique en cours menée par Mélanie Forné ("types-variétés » et «modal ») des collections de 40 sondages faits au centre et dans tous les GGR (sauf celui qui est situé hors de la 


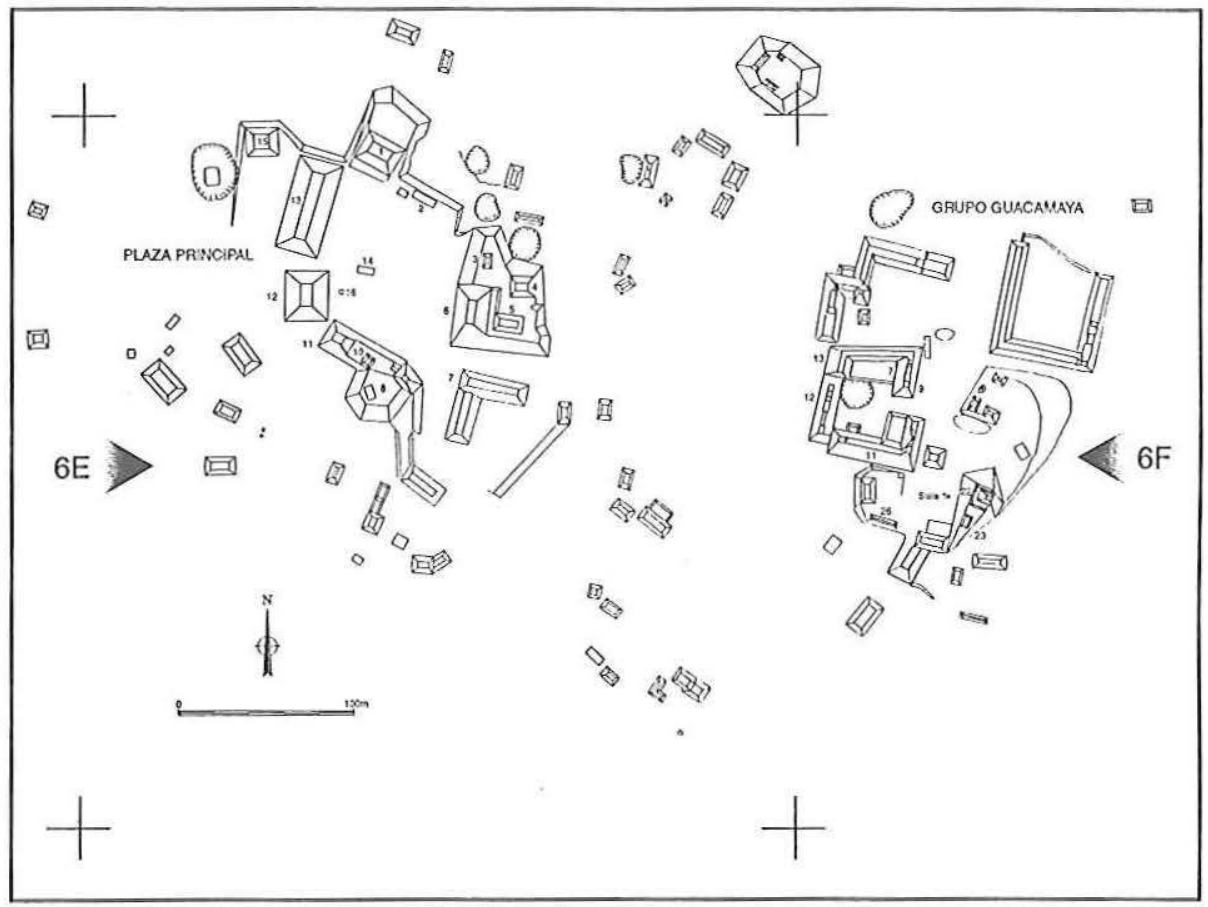

FIG. 3. - Plan préliminaire du centre de La Joyanca (quadrants $6 \mathrm{E}$ et $6 \mathrm{~F}$ )

zone protégée) et quelques-uns de classe III (programme réalisé par Martin Rangel, Laura Gamez, Diego Guerra et Raquel Macario) ; on dispose aussi d'une dizaine de datations radiocarbone faites par Nadine Tisnérat (CNRS, LSCE). La séquence d'occupation de La Joyanca débute dès le Préclassique moyen (800 av. J. C.) et se termine au Classique final (900 ou 1000 apr. J. C.) ${ }^{3}$. L'occupation la plus ancienne s'est étendue sur un arc sud, depuis le Groupe Gavilán à l'est jusqu'au Groupe Cojolita à l'ouest, en passant par le Groupe Guacamaya (Patio Sud) et la Place Principale (Figures 2 et 3) : cela est logique puisque cet arc forme le rebord de la meseta et donne accès au sibal (marais au pied de la cuesta), selon ce qui est observé sur les plateaux. Les sondages effectués dans les Patios Sud et Central de Guacamaya indiquent qu'une longue séquence de construction s'étend du Préclassique ${ }^{4}$ au Classique final ce que confirme la fouille, par Laura Gamez, d'une petite structure rituelle proche de la stèle 1 qui ne comporte pas moins de cinq édifices superposés. Plus au nord, les sondages effectués dans les GGR indiquent que ces groupes auraient été fondés au Classique récent, donc après le Groupe Guacamaya. Les quadrilatères fermés (Figure 2 : en carrés 5G, 4F et 4D), considérés comme les plus tardifs au Classique récent (V. Fialko, comm. pers. pour le nord-est du Petén), se trouvent de fait à la périphérie nord, ce qui tend à confirmer que l'établissement de La Joyanca s'est bien formé par extension du sud vers le nord.

Par conséquent, si le Groupe Guacamaya est le plus ancien des grands ensembles résidentiels de l'élite dans la cité, et aussi le plus complexe (de classe I), ses habitants 
furent probablement les responsables de la fondation de la Place Principale au Préclassique, puis de l'apogée que marquent en ce centre les édifices 6E-12 (ancien et récent) et $6 \mathrm{E}-13^{5}$. Car, en outre, c'est le groupe résidentiel le plus proche de la Place Principale et le seul qui a érigé une stèle au Classique ancien. Ses habitants appartenaient probablement au lignage qui aurait réussi à instaurer une dynastie gouvernante locale. D'autres groupes sociaux, probables lignages de l'élite, se seraient regroupés postérieurement de façon assez lâche au nord de la Place, fondant chacun un grand groupe résidentiel, qui s'est développé en patios multiples au Classique récent.

Postérieurement, le site de La Joyanca pourrait avoir été abandonné comme la plupart des sites des basses terres au $\mathrm{Ix}^{\mathrm{e}}$ siècle, voire au $\mathrm{x}^{\mathrm{e}}$ siècle, car la céramique Orange Fin est assez répandue sur le site. Une résidence d'un type peut-être non maya, connu au Classique final (850-1000) mais qui peut être postérieur, a été décapée en mars 2001 sur la Place Principale, dans un contexte stratigraphique qui suggère qu'il s'agit d'une réoccupation post-abandon. Certains palais ruinés du Groupe Guacamaya semblent aussi avoir été réoccupés après l'abandon, de façon fruste. Quoi qu'il en soit, la fin de la séquence d'occupation se situerait, d'après la céramique, au Classique final (900 ou 1000), car une occupation postclassique (1000-1524) n'est pas attestée à ce jour.

Les moments principaux de la longue séquence d'occupation de La Joyanca (d'une durée d'au moins 1500 ans) correspondent aux constructions publiques de la Place Principale ( $c f$. infra) et à la formation des grands groupes résidentiels fondés après le Groupe Guacamaya. Pour ces derniers, il s'agit d'obtenir des données comparatives concernant leur chronologie et leur architecture. Étant donné les dimensions de ces ensembles (les édifices des patios centraux n'ont pas moins de $40 \mathrm{~m}$ de long et 2 à $3 \mathrm{~m}$ de haut), un tel objectif est difficile à satisfaire. Indépendamment du programme déjà cité de sondages stratigraphiques appliqué à tous les groupes, Ernesto Arredondo a réalisé une série de tranchées exploratoires permettant de comparer, pour les patios centraux, tous les palais du côté sud à la hauteur de leur pièce centrale. En effet, les fouilles de dégagement de tous les édifices du Patio Central de Guacamaya, par Véronique Breuil-Martínez, tendaient à suggérer que cette pièce centrale de l'édifice sud est la mieux construite, qu'elle est dotée de la plus grande banquette maçonnée, face à une entrée relativement prestigieuse (escalier d'accès, façade moulurée, axe de symétrie..., Figure 4). Notre postulat est que tous les groupes de l'élite de La Joyanca ont été construits selon le même modèle général : en effet, tous sont orientés de même façon, les patios centraux sont de dimensions comparables et leurs entrées se trouvent à l'est (Figure 2).

Les tranchées exploratoires dans les édifices sud des patios centraux des GGR Tepescuintle, Venado, Loro Real et Armadillo ont mis au jour des palais voûtés comparables aux palais de Guacamaya, aux sols et aux murs stuqués et peints relativement bien conservés (parfois sur plus d'un mètre de hauteur), dont la pièce centrale est partout dotée d'une banquette maçonnée (Figure 4), soit latérale (Guacamaya, Tepescuintle, Loro Real), soit centrale ornée d'une niche sur sa base frontale (Venado, Armadillo). Les maçonneries n'atteignent cependant pas la qualité remarquable de celle du palais sud de Guacamaya, à l'exception notable du palais sud de Venado. Ce dernier est sans conteste plus monumental que celui de Guacamaya : excellente taille des pierres de parement, qualité du stuc peint en polychromie, épais- 

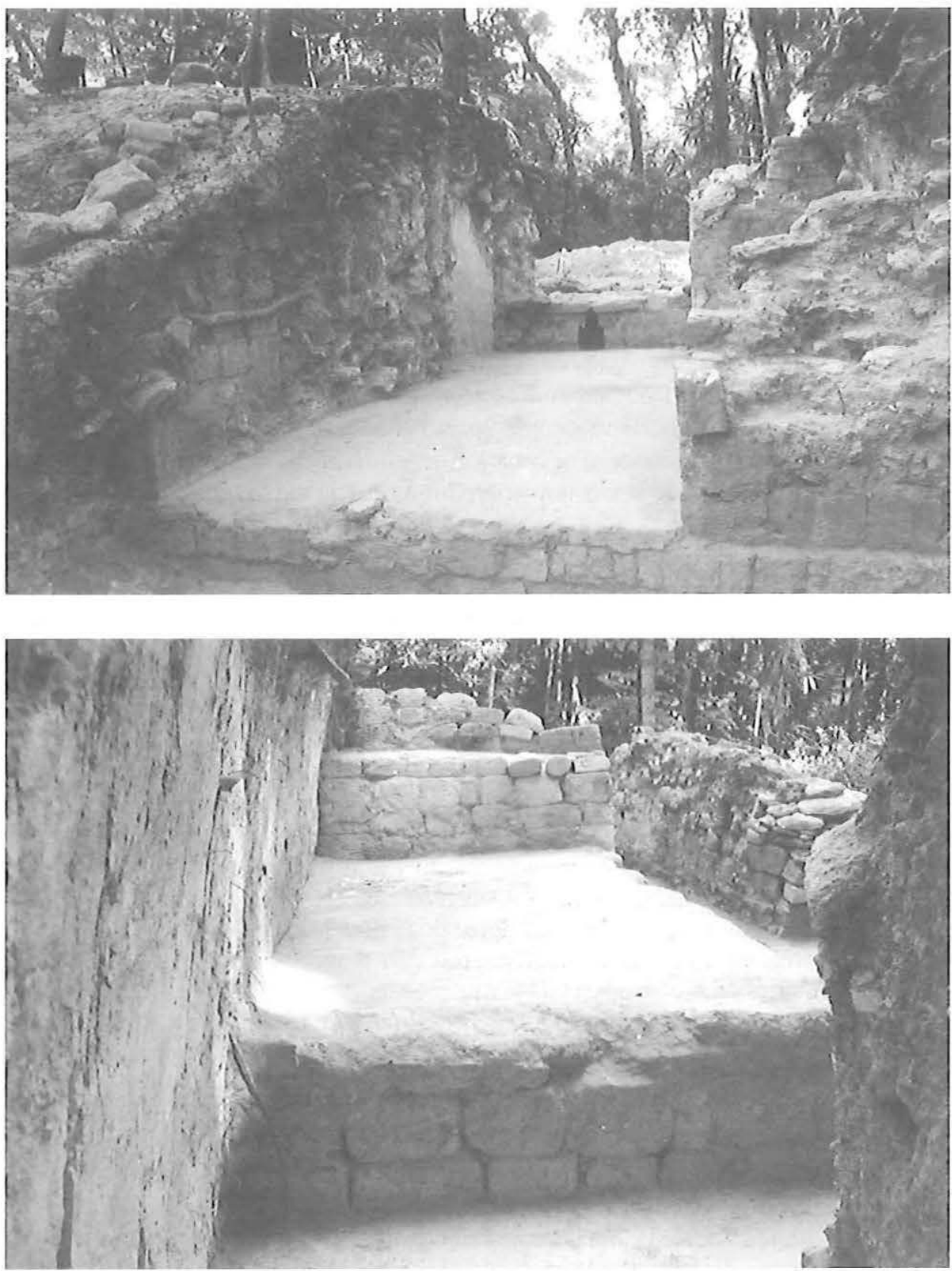

FIG. 4. - Vues des « palais » fouillés dans les Groupes Venado et Guacamaya 
seur des murs et surtout, antichambre précédant la pièce dotée de la banquette, dispositif qui tend à confirmer notre hypothèse que ces pièces centrales des édifices sud étaient des salles d'apparat ou de réception, au sein de chaque groupe (Figure 4).

Toutes ces données, bien qu'incomplètes encore, signifient que les grands groupes ont probablement été fondés au Classique récent (ou, au plus tôt, au Classique ancien, l'unique structure enfouie découverte, dans Venado, ayant donné un tesson ainsi daté), selon un modèle inventé dans le Patio Central de Guacamaya, et que leur occupation a été courte. Cependant, le palais sud du Patio Central de Venado a atteint un degré d'élaboration architecturale supérieur à celui de Guacamaya. Émulation entre toutes les familles cherchant à construire des palais prestigieux ? Rivalité entre les familles des Groupes Guacamaya et Venado ? Il faudrait pouvoir dater relativement la fondation des différents groupes pour mieux cerner cette interprétation.

\section{LE CENTRE RELIGIEUX ET POLITIQUE (Figure 3)}

Les fouilles de 1999 à 2001 - réalisées par Marie-Charlotte Arnauld et Erick Ponciano - ont confirmé nos identifications fonctionnelles des principaux bâtiments de la Place Principale, dédiés aux activités religieuses et politiques. Deux templespyramides, structures $6 \mathrm{E}-12$ et $6 \mathrm{E}-6$, occupent les côtés ouest et est. À l'ouest, le bâtiment $6 \mathrm{E}-13$, long de $62 \mathrm{~m}$ sur une plate-forme haute de $4 \mathrm{~m}$, ferme la Place. Il n'y a pour ainsi dire pas de structures domestiques sur la Place, excepté $6 \mathrm{E}-4$ et $6 \mathrm{E}-5$ situées à l'arrière de la pyramide $6 \mathrm{E}-6$, et $6 \mathrm{E}-14$ qui fut construite après l'abandon des grands édifices. Les types de maçonnerie apparaissent étonnamment diversifiés et la variation ne semble pas avoir une signification chronologique à l'intérieur du Classique. Il faut en déduire une certaine habileté des artisans à exploiter toutes les ressources locales en calcaire plus ou moins friable ou dur, et à profiter de diverses influences extérieures, comme la taille aiguë de pierres de parement dont on voit l'équivalent dans les ruines de El Perú, le grand site voisin (20 km à l'est) malheureusement jamais fouillé.

L'histoire de la Place Principale débute au Préclassique, d'après nos fouilles profondes assez limitées, par l'exploitation d'une petite carrière de pierre du côté ouest, dont le remblai a donné la date ${ }^{14} \mathrm{C}$ la plus ancienne (917 cal BC, cf. note 3$)$. Sept sols stuqués superposés la couvrent, l'édification de la Place a donc été longue. La grande Plate-Forme Sud, au remblai préclassique d'après la céramique, masque aussi une construction ancienne comprenant un escalier de grands blocs équarris érodés. Des murs également frustes ont été mis au jour sous les sols de la Place face au futur édifice $6 \mathrm{E}-13$.

De la période classique ancienne (300-600), marquée dans le Groupe Guacamaya voisin par l'érection de la stèle 1 (en 485) et des autels monolithes associés, on ne connaît pour ainsi dire rien dans la Place Principale. De façon générale au Petén, cette période est difficile à détecter car la céramique change de façon très progressive depuis le Préclassique et les édifices alors construits sont masqués par ceux du Classique récent. Tel est le cas à La Joyanca : du côté ouest de la Place, sous le sol stuqué du temple 6E-12-sub, deux sols antérieurs plus profonds témoignent d'une occupation de la période 400-600; et sous l'édifice politique 6E-13, une plate-forme 6E-13-sub, 

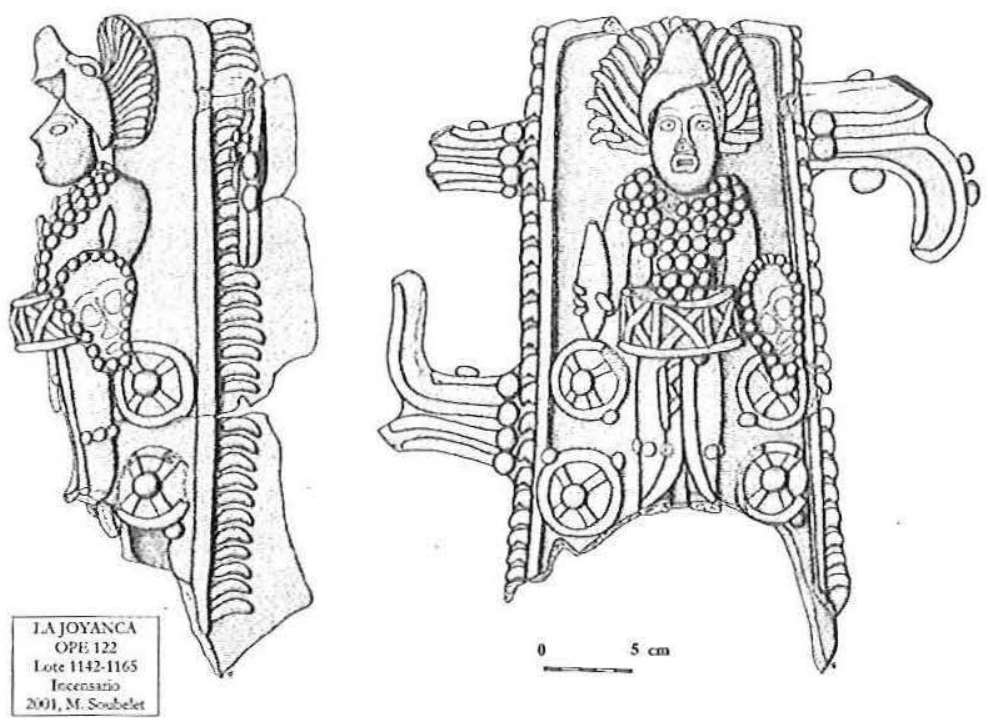

FIG. 5. - Encensoir trouvé sur le sol du temple 6E-6 (dessin : Marie Soubelet)

de 1 à $2 \mathrm{~m}$ de hauteur au revêtement stuqué peint en rouge, contenait une sépulture en ciste d'un individu peut-être de statut élevé, car accompagné de deux récipients céramiques polychromes à décors de faux glyphes, dont au moins un mode (base annulaire) est classique ancien ; une datation ${ }^{14} \mathrm{C}$ sera tentée et la fouille de cette plate-forme 13-sub sera amplifiée.

La période classique récente voit l'apogée de La Joyanca, c'est-à-dire de son centre politico-religieux, certainement encore du Groupe Guacamaya, ainsi que de la zone résidentielle formée par les GGR. Du côté est de la Place Principale, au bord nord de la grande allée qui mène au Groupe Guacamaya, a été construite la première pyramide-temple 6E-6 (646 cal AD, cf. note 5), haute d'environ $10 \mathrm{~m}$; mais nous ne savons rien des structures antérieures qu'elle recouvre probablement. Elle présentait trois corps, hauts chacun de $3 \mathrm{~m}$ à $3,40 \mathrm{~m}$, avec un grand escalier saillant sans rampe donnant sur la place, à la base duquel ont été trouvés les vestiges d'un décor de stuc modelé polychrome, associé à des fragments d'encensoirs, à de l'obsidienne et à du silex. Au sommet de 6E-6, le sol intérieur du temple maçonné et voûté a donné trois encensoirs modelés, dont un à décor anthropomorphe élaboré (Figure 5). Les deux maisons annexes à la pyramide, dont l'une d'excellente construction (6E-5), constituent le seul habitat directement associé aux édifices publics ; des vestiges domestiques jonchaient le sol et une sépulture en ciste a été fouillée sous le patio. Pour la plupart, les 21 sépultures fouillées à La Joyanca, dont l'étude par Véronique Gervais est en cours, ont été trouvées sous les patios domestiques ou, dans les palais, sous les banquettes.

En face de la pyramide 6E-6, apparemment au même moment qu'elle $(674$ cal $\mathrm{AD}^{6}$ ), fut édifié le petit temple 6E-12-sub. Il était peut-être dédié à un gouvernant ou à sa famille, car il présente à l'intérieur une figure de stuc polychrome anthropomorphe à masque solaire, debout de face, et un texte glyphique gravé qui mentionne peut-être ce personnage mais dont l'interprétation est difficile (Figure 6). Fait relati- 


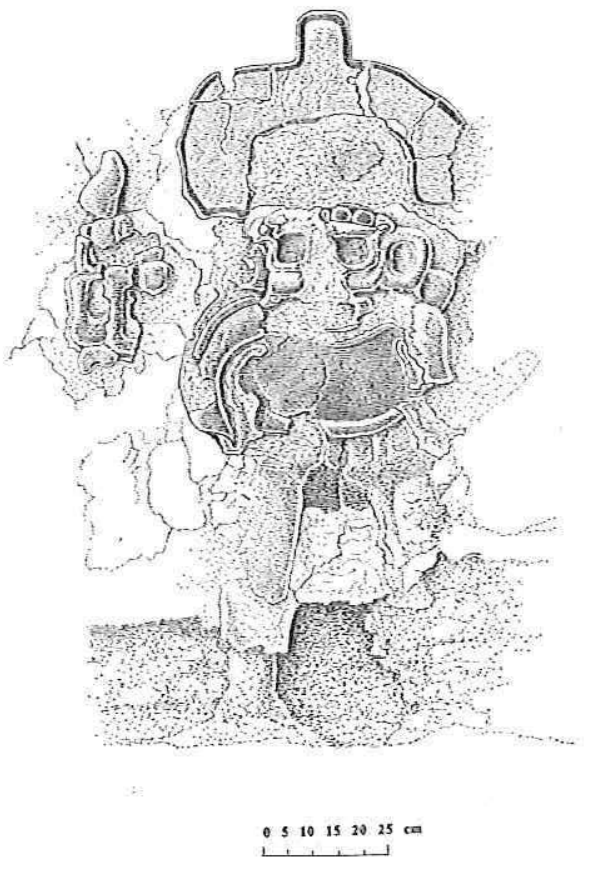

a

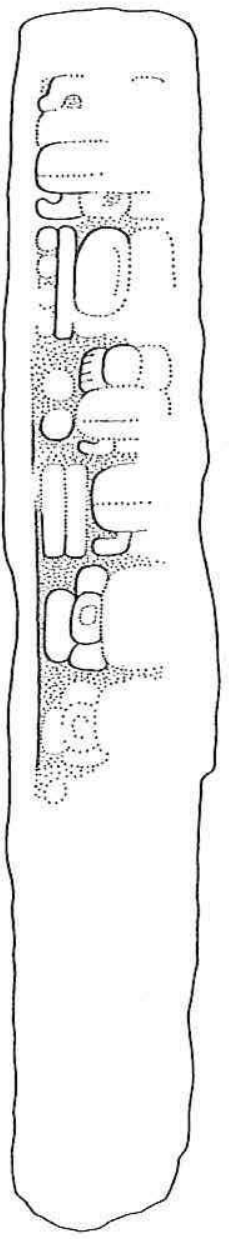

sud

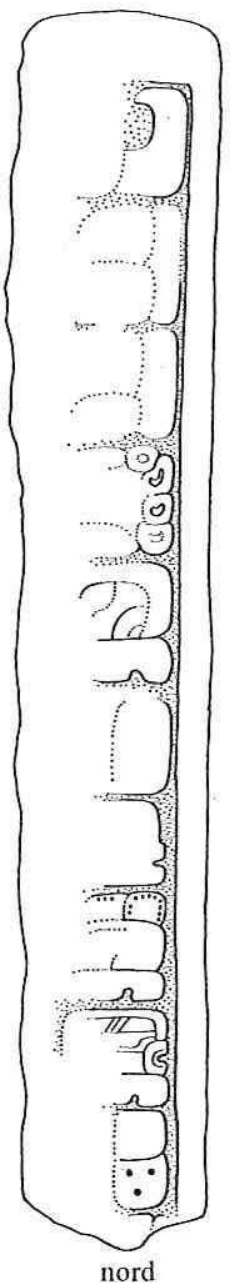

b

FIG. 6.

a : Figure de stuc modelé appliqué sur le mur intérieur du temple 6E-12-sub, face à l'entrée (dessin : Edgar Ortega)

b : Texte glyphique que porte la stèle 1 (chants nord et sud), Groupe Guacamaya, La Joyanca (dessin : David Stuart)

vement exceptionnel, ce temple est conservé entier, de sa plate-forme jusqu'à sa crête faîtière, et ses trois pièces gardent leurs voûtes en encorbellement. Mais le décor de stuc modelé qui ornait à l'extérieur le dessus de la porte d'entrée a été détruit lors du remblaiement de l'édifice pour la construction de la pyramide-temple postérieure. Car, dans la deuxième moitié du Classique récent (750-850), eut lieu un grand épisode de construction qui forma tout le côté ouest de la Place où furent accolés le temple 6E-12, sur un soubassement pyramidal à six degrés de $10 \mathrm{~m}$ de hauteur totale (selon les 
restitutions de Tristan Saint-Dizier) couvrant le petit temple 6E-12-sub, et le grand édifice 6E-13 construit sur l'ancienne plate-forme 6E-13-sub. Les deux bâtiments voûtés sont d'assez bonne construction, encore que les meilleures pierres taillées aient été réservées au temple 6E-12. Comme le temple 6E-6, qui lui fait face à l'est, ce dernier ne comportait qu'une pièce étroite et sur son sol intérieur stuqué gisaient des fragments d'encensoirs et deux tambours de pierre taillée, dont un orné de deux bandes de glyphes en bas-relief. Ce texte est le troisième qui nous soit connu à La Joyanca, avec la stèle 1 du Groupe Guacamaya et le texte gravé dans le temple ancien 6E-12-sub.

Si nos données de 2001 sont exactes (fouilles non terminées), l'édifice 6E-13 comportait à l'origine trois grandes salles en ligne d'environ $16 \mathrm{~m}$ de long chacune, à trois portes chacune, soit neuf portes, chiffre symbolique ; le grand escalier d'accès a aussi neuf marches (Figure 7). Il semble que seule la pièce centrale soit dotée d'une banquette centrale. Or, même si formellement il ressemble à un palais, ce bâtiment n'a rien d'une résidence, car aucun matériel domestique ne lui est associé ; on a par contre trouvé sur la première marche de l'escalier, dans l'axe de la pièce centrale, un objet (rare au Classique) plutôt associé aux fonctions publiques : un sceau de céramique. Bref, 6E-13, la plus grande structure de La Joyanca, appartient assez probablement à la catégorie des édifices dits « sièges du pouvoir » (Michelet \& Becquelin 1995). Cela étant, les trois salles juxtaposées sont exceptionnellement longues et évoquent une autre catégorie de bâtiment public maya, la « maison longue » (>15-18 m), qui servait avant tout de salle de réunion aux hommes de la communauté (Arnauld 2001). La pièce à banquette centrale aurait abrité les audiences d'un gouvernant (Kowalski 1987, chap. VI). Si les données se confirment, l'édifice public $6 \mathrm{E}-13$ pourrait ainsi refléter une forme de gouvernement combinant un pouvoir fort au sommet avec une institution réunissant des hommes de la communauté. En d'autres termes, ce système aurait associé un gouvernant de la famille du Groupe Guacamaya - auquel étaient peut-être dédiés les temples 6E-12 et 12-sub - et les chefs des principaux lignages des grands groupes résidentiels fondés au Classique récent au nord de la Place Principale ${ }^{7}$.

Or il est intéressant d'observer l'évolution de l'édifice 6E-13 après sa construction. Deux des salles, dont la centrale, ont été subdivisées en plusieurs étapes pour former finalement trois pièces de plus, ne laissant comme grand espace de " réunion » que la salle nord de $16,25 \mathrm{~m}$ et la salle sud de $10,75 \mathrm{~m}$. Dans la petite pièce centrale, la banquette a finalement été dépouillée de son parement de pierres taillées, son remblai étant laissé à nu. Puis la voûte de l'édifice s'est effondrée et, à côté de la banquette, sur les décombres remaniés, a été déposé sans ménagement un individu, en position atypique, couvert ensuite de pierres (Sép. 11, individu de sexe féminin selon V. Gervais). La stratification exclut que l'individu ait été tué par l'effondrement du toit et rend peu probable qu'il ait été placé par hasard au bord de la banquette centrale détruite ; mais rien ne prouve que l'effondrement et la « sépulture » soient contemporains (on tentera de dater les ossements). Ces différents événements, non dénués de violence, suggèrent que la fin de l'occupation de l'édifice politique $6 \mathrm{E}-13$ a été marquée par des conflits, sans qu'il y ait pour autant trace de guerre. Doit-on relier ces conflits à cette forme de rivalité entre lignages qui se dégage de l'étude préliminaire de l'architecture des grands groupes résidentiels? 


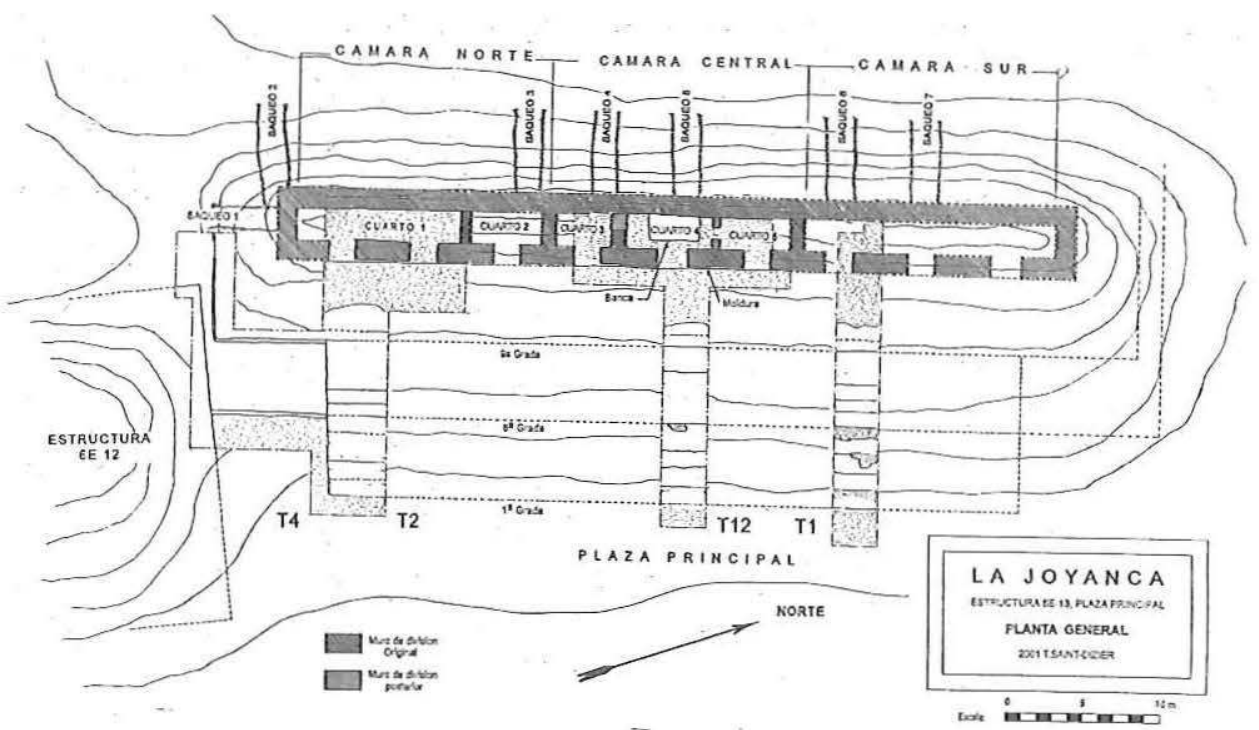

FIG. 7. - Plan de la structure 6E-13, Place Principale, La Joyanca (relevé et dessin : Tristan Saint-Dizier)

Après l'abandon de la fin du Classique récent, la maison tardive $6 \mathrm{E}-14$ trouvée sur la Place fut construite en partie avec les pierres taillées récupérées sur les édifices publics $6 \mathrm{E}-13$ et $6 \mathrm{E}-12$ abandonnés.

\section{HABITAT DOMESTIQUE ET MILIEU ENVIRONNANT}

De même que nous tentons de comprendre les relations entre les occupants du Groupe Guacamaya et ceux des GGR de classe II, de même une approche essentielle de l'organisation socio-politique de La Joyanca consiste à tenter de restituer, par l'étude des structures de l'habitat, les relations entre les groupes de classe II et les groupes de classe III, dans cet établissement dont la densité est assez basse. En d'autres termes, il s'agit d'évaluer les quantités et les densités de petits monticules autour des patios à palais (par exemple, la plus grande concentration est bien associée au Groupe Guacamaya) en considérant que ces hypothétiques ensembles sociaux partageaient des ressources locales, c'est-à-dire, pour ce qui laisse des traces en surface, des carrières de pierres et des réservoirs d'eau de pluie - que nous réutilisons pour les consolidations des structures dégagées. Notre objectif, commun avec l'équipe des géographes que dirige Jean-Paul Métailié, consiste à évaluer le caractère plus rural que vraiment urbain de l'établissement de La Joyanca, postulant que ses groupes résidentiels de classes III, et même II, auraient été imbriqués dans un parcellaire horticole local constitué sur les sols fertiles relativement épais du plateau (rendzines, jusqu'à $40 \mathrm{~cm}$ d'épaisseur). Il s'agit en bref de restituer les structures de l'habitat dans leur milieu environnant modifié par l'homme. Dans cette perspective, Dominique Michelet fait le relevé topographique précis du centre (2001) et des GGR (2002) et Éva Lemonnier mène non seulement des prospections systématiques dans la zone résidentielle, mais 
aussi des fouilles extensives et intensives dans le Groupe Gavilán, qui fait partie de la concentration de classe IIIA du Groupe Guacamaya au nord-ouest (Figure 2).

Les décapages effectués sur les monticules bas $(<50 \mathrm{~cm})$ du Groupe Gavilán ont mis au jour des maisons à deux pièces, sans banquette, dont les murs reposaient sur des bases de pierres et dont les sols étaient stuqués. Plusieurs dépotoirs ont été fouillés, fournissant un important matériel lithique (silex, obsidienne) analysé par Sébastien Lacombe. Les sols extérieurs, étudiés par séries de sondages en transects et en damiers sur environ $2500 \mathrm{~m}^{2}$, révèlent des paléo-sols superposés bien conservés. L'emplacement a connu quatre étapes d'occupation, avec une concentration de sépultures sous un sol extérieur stuqué associé à une structure circulaire maçonnée. Cette séquence a considérablement modifié le milieu. Plusieurs échantillonnages des paléo-sols extérieurs donnent lieu à des séries d'analyses pédo-sédimentologiques en cours et, selon une approche géo-archéologique, des sondages ont aussi été faits par le sédimentologue Jean-Michel Carozza en vue de la description et de la datation des paléo-sols les mieux conservés, dont l'un est antérieur à l'occupation. En outre, des tranchées pratiquées à proximité du Groupe Gavilán dans des paléo-chenaux karstiques indiquent une forte sédimentation dans les micro-reliefs du plateau, comportant de la céramique depuis la base. La stratification d'une tranchée faite par le géographe Jean-Paul Métailié au pied du plateau, au contact avec le marais autrefois plus étendu, montre que le phénomène de colluvionnement a été réel (avancée des dépôts terrigènes sur la zone du marais), qu'il date bien de l'occupation humaine du site, mais qu'il a été assez limité ( $60 \mathrm{~cm}$ d'épaisseur maximale), indiquant une dégradation moyenne des sols du plateau. Toutes ces données suggèrent que le plateau a pu être mis en culture dans l'établissement même, mais que les seules activités domestiques et architecturales peuvent tout autant expliquer la sédimentation.

Au-delà des objectifs liés à l'archéologie de l'habitat ancien, les études géographiques, visent à restituer l'histoire du milieu forestier et des paysages. Un sondage de $3 \mathrm{~m}$ de profondeur effectué en mars 2000 dans le lac Tuspán sous la direction du palynologue Didier Galop, au débouché ouest du marais permanent qui borde le plateau de La Joyanca, a fourni trois colonnes actuellement en cours d'analyse (pollen, sédiments et mollusques). Les premières données polliniques indiquent que, de -115 à $-300 \mathrm{~cm}$, les niveaux comportent des concentrations polliniques suffïsantes, avec du maïs présent dès $-295 \mathrm{~cm}$ dans un milieu forestier déjà dégradé ; plusieurs phases culturales suivent, avec un approfondissement du lac $(-210 \mathrm{~cm})$ jusqu'à une reprise de la forêt semi-sempervirente (à partir de $-150,-155 \mathrm{~cm}$ ) ; cette phase de reforestation à la fin du Préclassique et au début du Classique - datation ${ }^{14} \mathrm{C}$ à $-115 \mathrm{~cm}: 1710 \pm 40 \mathrm{BP}$, Beta 153910, 240 (350) 420 cal AD — a été repérée ailleurs au Petén (Dunning et al. 1997, 1998). Bien que difficile à dater (effet d'« eau dure »), cette séquence permet de reconstituer sur près de $200 \mathrm{~cm}$ d'épaisseur une histoire durant laquelle on peut suivre l'évolution des paysages et des activités agraires au cours du Préclassique.

Parce que l'histoire de l'environnement s'appuie sur une connaissance du milieu actuel, la troisième composante des études géographiques du projet concerne les dynamiques récentes et actuelles, au moyen d'enquêtes de terrain et par télédétection, études faites par J.-P. Métailié et Gilles Selleron. Les premiers défrichements à vocation agricole dans la zone datent de 1991 le long du lac Tuspán. Dix ans plus tard, il 
existe cinq communautés qui représentent une population de 1100 personnes environ, soit une densité d'à peine 5 habitants $/ \mathrm{km}^{2}$ (surfaces non cultivables déduites). L'impact des incendies non contrôlés est sans commune mesure avec celui des simples défrichements et apparaît comme un des facteurs aujourd'hui fondamentaux de l'évolution des milieux, un seuil ayant probablement été franchi entre 1998 et 2001 (sécheresse de l'épisode Niño). Cet état de fait oriente inexorablement les choix productifs sur des espèces à cycles cours et accélère l'activité d'extraction du bois. Par ailleurs, l'étude des dynamiques de construction des territoires issus de la colonisation, montre que deux grandes logiques sont à l'œuvre : le système d'appropriation du territoire par les Indiens q'eqchi suit une logique ancestrale de milpero (cultivateur de maïs), supposant la recherche des meilleures terres pour la culture du maïs. De son côté, le système ladino, où l'élevage bovin est prestigieux, s'organise à partir de terres aptes au pâturage. Cette représentation un peu manichéenne de la dynamique territoriale de la frontière devra être nuancée, à l'heure où la saturation de l'espace engendre des comportements d'opportunisme et d'innovation et où se raréfie la ressource la plus convoitée, qui est ici la forêt haute des plateaux, milieu équivalent à celui du site archéologique de La Joyanca.

\section{Conclusion}

Ce site de La Joyanca apporte des éléments nouveaux dans le corpus des " cités » mayas classiques des basses terres du Petén : désintégration du centre politicoreligieux et des ensembles de palais, association étroite au centre, dans le temps et dans l'espace, des lieux de culte et d'un grand édifice politique complexe et "évolutif », récurrence d'un modèle d'ensemble résidentiel à fort investissement architectural, faible densité de l'habitat, probable mise en culture de l'établissement même... Nos reconnaissances des plateaux demeurent insuffisantes à le prouver, mais il semble bien que l'habitat dispersé y soit aussi structuré par la répétition de grands groupes résidentiels équivalents à ceux de La Joyanca. Si cette hypothèse se confirme, on pourrait proposer un modèle d'organisation locale hiérarchisée à trois rangs, dont le centre, La Joyanca, aurait été constitué progressivement par la famille la plus anciennement installée, attirant autour d'elle une partie de l'élite régionale susceptible de l'aider à doter la petite cité des éléments essentiels que sont les temples-pyramides avec décors stuqués, l'édifice " siège du pouvoir », les textes glyphiques... Il est intéressant de remarquer que tous ces éléments - ceux que nous sommes en mesure d'étudier représentent des productions moyennement élaborées (à l'exception de certaines maçonneries), qui seraient bien le fait d'élites locales quelque peu marginales par rapport aux grands centres des sociétés mayas classiques. En quelque sorte, on aurait là un mode d'organisation élémentaire et intermédiaire des « campagnes » au Petén nord-ouest ${ }^{8}$.

\section{NOTES}

1. Le projet PNO-La Joyanca a été formulé en 1998 pour quatre années, de 1999 à 2002. Approuvé par l'Institut d'Anthropologie et d'Histoire du Guatemala (IDAEH), il s'inscrit dans le cadre des recherches de 
l'antenne du CEMCA au Guatemala. Le financement est assuré par la compagnie Basic Resources International (Bahamas) Ltd. Sucursal Guatemala, le ministère des Affaires étrangères et le CNRS, dont le PEVS (Programme " Environnement, Vie et Sociétés », comité « Histoire des interactions société-nature »). Les chercheurs français appartiennent à l'UMR 8096: Archéologie des Amériques (Nanterre), à l'UMR 5602: GEODE Géographie de l'environnement (Toulouse), à l'UMR 9946 : Chrono-écologie du Quaternaire (Besançon) à l'UMR 1572: LSCE, Laboratoire des sciences du climat et de l'environnement (Gif-sur-Yvette) et à l'UMR 7007: CEREG, Centre d'Études et de Recherches écogéographiques (Strasbourg); les chercheurs guatémaltèques appartiennent à l'université de San Carlos et à l'université del Valle. Les analyses de malacologie sont effectuées à la Sub-Dirección de Laboratorios y Apoyo Académico (INAH), Mexique.

2. En aval, les autres sites importants de la région, Mactún et La Florida par exemple, sont bien situés quasiment sur la rive du fleuve. Par contre, en amont, le très grand site de El Perú est situé à une heure du fleuve, accessible par un petit affluent actuellement envasé. Il est difficile de comprendre à l'heure actuelle l'implantation des grandes cités mayas par rapport aux deux grands systèmes fluviaux qui drainent les flancs est et ouest de la partie centrale des basses terres, c'est-à-dire le "système Belize" à l'est et celui de l'Usumacinta (avec le San Pedro parmi ses aflluents) et du Candelaria à l'ouest. La circulation par voies d'eau à l'époque pré-hispanique demeure à peu près inconnue dans les basses terres.

3. Les dates les plus anciennes sont : $2780 \pm 70 \mathrm{BP}$ (GifA $100621 ; 1119$ (917) $808 \mathrm{cal} \mathrm{BC}, 2$ sigma) pour le remblai d'une ancienne carrière de pierres de la Place Principale, et $2520 \pm 70 \mathrm{BP}$ (GifA 100628;800 $(763,676,674) 415$ cal BC, 2 sigma) pour un paléo-sol sous la structure 6E-13 de la même Place. La dernière occupation est représentée par la str. $6 \mathrm{E}-14$ de la Place Principale, dont la morphologie en forme de " $\mathrm{C}$ » (Rice 1986) est datée du Classique Final (850-1000).

4. Trois dates pour trois échantillons stratifiés d'un même sondage dans le Patio Sud : $2470 \pm 70 \mathrm{BP}$, GifA $100626: 770(757,695,541) 409 \mathrm{cal} \mathrm{BC}, 2$ sigma ; $2110 \pm 70$ BP (GifA 100625; $358(161,130,120)$ cal $\mathrm{BC}-22$ cal AD, 2 sigma, et $2000 \pm 60 \mathrm{BP}$, GifA $100624: 161$ cal BC $(2,14,16) 124$ cal AD, 2 sigma.

5. Deux dates ${ }^{14} \mathrm{C}$ attestent la contemporanéité du Groupe Guacamaya et de la Place Principale aux époques anciennes : $2470 \pm 70 \mathrm{BP}$ déjà citée (GifA 100626) pour le Patio Sud et $2520 \pm 70 \mathrm{BP}$ déjà citée (GifA 100628) pour un paléo-sol de la Place; ainsi qu'à l'époque classique récente » : $1300 \pm 60 \mathrm{BP}$ (GifA 100627 ; 646 (689) 877 cal AD, 2 sigma) pour le sol d'un palais du Patio Central du Groupe Guacamaya et $1410 \pm 60 \mathrm{BP}$ (GifA $100629 ; 534$ (646) $769 \mathrm{cal}$ AD, 2 sigma) pour un autel au pied de la pyramide $6 \mathrm{E}-6$ de la Place Principale.

6. $1330 \pm 70$ BP (GifA 100622; 603 (674) 875 cal AD, 2 sigma); une deuxième date obtenue sur un échantillon provenant de la couche sous-jacente au sol intérieur nous parait un peu tardive : $1230 \pm 60 \mathrm{BP}$ (GifA 100620, 671 (778) $950 \mathrm{cal} \mathrm{AD}, 2$ sigma). La contemporanéité des temples 6E-6 à l'est et 6E-12-sub à l'ouest est surprenante dans la mesure où l'oriental repose sur une pyramide haute de près de $10 \mathrm{~m}$ alors que l'occidental se trouve sur une plate-forme de $1,50 \mathrm{~m}$ de hauteur; la symétrie n'est cependant pas une nécessité. Les similitudes apparentes entre leurs décors de stuc modelé polychrome (6E-6: en bas sur l'escalier ; 6E-12-sub : au-dessus de la porte d'entrée et dans le couloir d'entrée) seraient en accord avec la contemporanéité, que nous tenterons de confirmer en datant le charbon associé au stuc modelé de l'escalier de $6 \mathrm{E}-6$.

7. Cette hypothèse est impossible à démontrer mais pourrait acquérir quelque validité par le moyen d'analyses comparatives, si des structures longues hors palais des centres mayas classiques d'un rang équivalent à La Joyanca étaient fouillées. En réalité, les bâtiments publics en dehors des complexes de palais comme dans le cas de La Joyanca - dont c'est l'un des grands intérêts - , ou bien n'existent pas dans les sites moyens du Petén, ou bien ne sont pas reconnus (Ball \& Taschek 1991, note 9). La catégorie d'édifice « siège du pouvoir » a d'ailleurs été définie hors du Petén dans les basses terres du nord (Michelet \& Becquelin 1995) et celle de « maison longue » est à peine reconnue pour le Classique (Arnauld 2001).

8. Une saison de travail de terrain sera réalisée en 2002 et une dernière campagne devrait permettre en 2003 de compléter les consolidations d'édifices commencées en 2000, et effectuées sous la direction de Enrique Monterroso. Pour leur appui constant et leur confiance, nous souhaitons remercier Mmes et MM. Juan Antonio Valdés, Elizabeth Lemus, Anaité Galeotti, Luis Castillo, Guillermo Diaz, Nora López, Edgar Ortega et Pedro Córdoba (Institut d'anthropologie et d'histoire du Guatemala), Gilberte Beaux (Basic Holdings Ltd), Rodolfo Sosa et Antonio Minondo (Basic Resources), Martine Dauzier et Véronique Gervais et Jérôme Monnet (CEMCA), ainsi que de nombreux collègues guatémaltèques, français, espagnols et américains qui nous ont fait partager leurs données ou leur expérience, en particulier ceux des projets Piedras Negras, Balamkú, Aguateca, Colonial, « Atlas Arqueológico » et Yaxha-Nakum-Naranjo. Notre reconnaissance va enfin aux 65 hommes des quatre communautés voisines de colons, El Tambo, La Esperanza, 17 de Abril et El Aguacate, qui travaillent avec nous à La Joyanca et protègent le site et le campement. 


\section{RÉFÉRENCES BIBLIOGRAPHIQUES}

ArNauld, M.-C., 2001. - « La "casa grande": evolución de la arquitectura del poder del Clásico al Postclásico », in : A. Ciudad R., M. J. Iglesias \& C. Martinez (ed.), Reconstruyendo la ciudad maya : el urbanismo en las sociedades antiguas, pp. 363-401, Sociedad Española de Estudios Mayas, Madrid.

Arnauld, M.-C., E. Ponciano \& V. Breull-Martinez (ed.), 2000. - Proyecto Petén Noroccidente-La Joyanca, Informe $n^{o} 2$, segunda temporada de campo, 2000, CEMCA, Guatemala (359 p., 190 ill.).

Arnauld, M.-C. \& P. I. Morales (ed.). 1999. - Proyecto Petén Noroccidente-La Joyanca, Informe $n^{\circ}$ 1, Primera temporada de campo, 1999, CEMCA, Guatemala (153 p., 64 ill.).

Arnauld, M.-C., D. Michelet, F. de Pierrebourg, P. Nondédéo \& G. Pereira, 1999. "Balamkú : tercera temporada de campo (1998) », in : J.-P. Laporte \& H. L. Escobedo (ed.), XII Simposio de Investigaciones Arqueológicas en Guatemala, pp. 695-710, Museo Nacional de Arqueología y Etnología de Guatemala, Asociación Tikal, Guatemala.

BALL, J. W. \& J. T. TASCHEK, 1991. — « Late Classic Lowland Maya political organization and central-place analysis. New Insights from the Upper Belize Valley ", Ancient Mesoamerica, 2, pp. 149-165.

Berlin, H., 1958. — « El glifo “emblema ” en las inscripciones mayas », Journal de la Société des Américanistes, XLVII, pp. 111-119.

Culbert, T. P. \& Rice, D. S., 1990. - Precolumbian population history in the Maya lowlands, University of New Mexico Press, Albuquerque.

Demarest, A. A., 1997. - "The Vanderbilt Petexbatun Regional Archaeological Project 1989-1994: Overview, History and Major Results of a Multidisciplinary Study of the Classic Maya Collapse ", Ancient Mesoamerica, 8 (2), pp. 207-364.

DrenNan, R. D., 1988. - « Household location and compact versus dispersed settlement in Prehispanic Mesoamerica ", in : R. R. Wilk \& W. Ashmore (ed.), Maya household and community in the past, pp. 250-273, University of New Mexico Press, Albuquerque.

Dunning, N. P., T. BeACH \& D. Rue, 1997. — « The paleoecology and ancient settlement of the Petexbatun region, Guatemala », Ancient Mesoamerica, 8, pp. 255-266.

Dunning, N. P., D. Rue, T. Beach, A. Covich \& A. Traverse, 1998. — « Human-environment interactions in a tropical watershed : the paleoecology of Laguna Tamarindito, El Petén, Guatemala », Journal of Field Archaeology, 25, pp. 139-151.

Escobedo, H. L. \& S. D. Houston, 1999. - Proyecto Arqueológico Piedras Negras, Informe preliminar $n^{\circ} 3$, Tercera temporada, 1999, Universidad de Brigham Young-Universidad del Valle, Guatemala.

Graham, I., 1988. — « Homeless hieroglyphs », Antiquity, 62, pp. 122-126.

Graham, I. \& E. Von Euw, 1982. - Corpus of Maya Hieroglyphic Inscriptions, vol. III, Part 3 : Yaxchilan, Peabody Museum of Archaeology and Ethnology, Harvard University, Cambridge.

Kowalski, J., 1987. - The House of the Governor : A Maya Palace of Uxmal, Yucatan, Mexico, University of Oklahoma Press, Norman. 
Mathews, P., 1985. - « Maya Early Classic Monuments and inscriptions », in : G. R. Willey \& P. Mathews (ed.), A consideration of the Early Classic period in the Maya Lowlands, pp. 5-54, Institute for Mesoamerican Studies, State University of New York at Albany Publication $\mathrm{n}^{\circ} 10$, Albany.

Michelet D., M.-C. Arnauld, P. Becquelin, M.-F. Fauvet-Berthelot, P. Nondédéo, F. de Pierrebourg \& É. Taladoire, 1997. - « Le Groupe Sud de Balamkú (Campeche, Mexique) : éléments d'une histoire architecturale mouvementée », Journal de la Société des Américanistes, LxxxIII, pp. 229-249.

Michelet D., M.-C. Arnauld, P. Nondédéo, G. Pereira, F. de Pierrebourg \& É. Taladoire, 1998. - " La saison de fouilles de 1998 à Balamkú (Campeche, Mexique) : des avancées substantielles ", Journal de la Société des Américanistes, Lxxxiv (1), pp. 183-199.

Michelet D. \& P. Becquelin, 1995. - «Elementos políticos y religiosos de un sector de la región Puuc, su identificación e interpretación », in : C. Varela, J.-L. Bonor y Y. Fernández (ed.), Religión y sociedad en el área maya, pp. 109-134, Cuarta Mesa Redonda de la Sociedad Española de Estudios Mayas, Madrid.

Nondédéo, P., 2001. - Évolution des sites mayas dans le sud-est de l'État du Campeche, Mexique, thèse de doctorat, université de Paris I-Panthéon Sorbonne, Paris.

Reents, D. \& R. Bishop, 1987. - « The Classic Maya " Codex Style ”Pottery ", in : Memorias del Primer Coloquio Internacional de Mayistas, pp. 775-788, Universidad Nacional Autónoma de México, México.

Rice, D., 1986. - «The Petén Postclassic : A Settlement Perspective », in : J. A. Sabloff \& E. W. Andrews V (ed.), Late Lowland Maya Civilization : Classic to Postclassic, pp. 301-346, University of New Mexico Press, Albuquerque.

Robicsex, F. \& D. M. Hales, 1981. - The Maya Book of the Dead: The Ceramic Codex, The Maya Publishing Company, Charlotte, North Carolina.

Simon, M. \& N. Grube, 1995. — « Maya superstates », Archaeology (nov-dec.), pp. 41-46. 